\title{
Force Control for Robotic-Assisted Surgery Based on Viscoelastic Tissue Model
}

\author{
Pedro Moreira ${ }^{*} \quad$ Chao Liu $^{*} \quad$ Nabil Zemiti ${ }^{*} \quad$ Philippe Poignet ${ }^{*}$ \\ (*)Université Montpellier 2, LIRMM, France \\ E-mail:pedro.moreira@lirmm.fr,liu@lirmm.fr,zemiti@lirmm.fr,poignet@lirmm.fr
}

\begin{abstract}
Usually, force control algorithms developed for medical applications use a basic elastic model to represent the interaction between the robot and soft tissue. It is known that soft tissues are complex structures with viscoelastic properties as well as nonheterogeneity and hysteresis behavior.

This work intends to improve the performance and the robustness of a force control scheme using a more realistic interaction model. The proposed force control is a model based algorithm using a Kelvin-Boltzmann viscoelastic model as the interaction model.
\end{abstract}

\section{Introduction}

Understanding the interaction between medical tools and living tissues has become a very important aspect as the use of robot in medical application increases. Medical robots can work together with surgeons helping to create a less invasive and more precise surgery. The need of better technologies for surgery is illustrated by the great number of research running all over the world on how robots can help on procedures such as interventional radiology and microsurgery.

In all those procedures the robot or the medical tool guided by the robot needs to get in contact with several types of soft tissues. In order to avoid tissue damage, the contact force and the tissue deformation should be precisely controlled.

One of the first works applying force control to medical robots is presented in [3], where the force feedback was used to provide safety, tactile capabilities and improve man/machine interface. More recently, force control has been studied to implement teleoperational systems with haptic feedback [4], and also to reject disturbances forces caused by physiological motions [5]. In this last application, the use of classical force controllers may not achieve a good disturbance rejection, mainly because of its bandwidth limitation [6]. Hence, increase the performance of force controllers in contact with soft tissue can significantly improve the use of robots in medical procedures.

The force control scheme presented in [7] and classical force controllers use either no interaction model or an elastic spring as interaction model. It is clear that purely elastic models cannot describe accurately the interaction between the robot and the tissue, especially when fast movements are involved, when the viscous characteristic of the tissue has a strong influence. Therefore, it is necessary to introduce a more realistic model in order to achieve a good disturbances rejection.

The objective of this work is to propose a force control algorithm to improve the contact between the robot and the patient in order to precisely control the tissue deformation. This control algorithm must reject all kinds of disturbances, mainly caused by physiological motions.

\section{Soft tissue modeling}

Three classical viscoelastic models commonly used and the elastic one were compared in order to define the best model for control applications. They are presented in Fig. 1. The Kelvin-Voigt model is composed by one spring and one damper in parallel, its equation for force is dependent on the deformation and the velocity of the deformation. For the Kelvin-Boltzmann model a spring is added in series with the Kelvin-Voigt model, in this case the derivative of the force appears in the force equation.

Different types of experiments can be performed in order to define soft tissue characteristics, applying axial forces and/or torsion on the tissue [1]. To define the best model, relaxations tests were performed, which consist in performing a position step input on 
the soft tissue and measuring the exerted force. In this work ex-vivo tests (piece of beef) were performed.

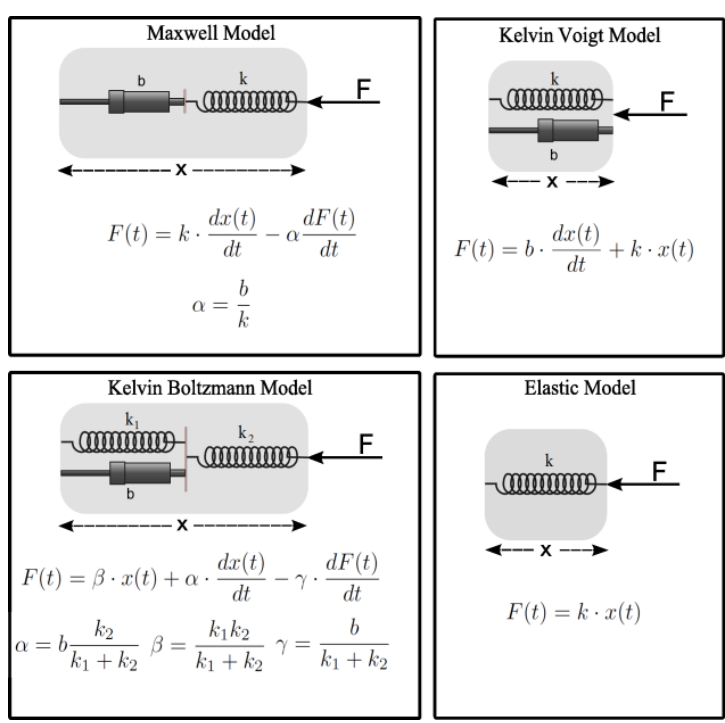

Figure 1: Elastic and viscoelastic model based on springs and dampers

All the data were collected and the parameters for each model were estimated using a Least Square algorithm. The estimated models were simulated using the input information collected and the output compared with the measured force. The results can be seen in Fig. 2. The parameters estimated for each model can be seen in Table 1 .
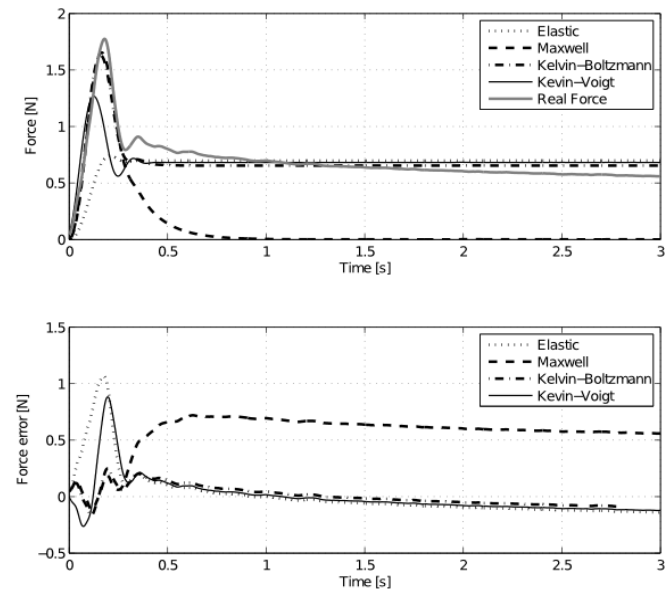

Figure 2: Relaxation tests: Measured force and simulated models

According to the performed tests, among the viscoelastic models, the Kelvin-Boltzmann is the one that presents the most realistic results with a dynamic response closer to the real one. This can be explained by the presence of the force derivative, which brings more information about the transient period. The Kelvin-Boltzmann equation is written as

$$
F_{e}=\gamma x+\beta \dot{x}-\alpha \dot{F}_{e}
$$

\begin{tabular}{|c|l|c|c|}
\hline Model & Parameters & Values & Stand. Dev. \\
\hline Elastic & $k$ & 107.28 & $0.58 \%$ \\
\hline \multirow{2}{*}{ Maxwell } & $\alpha$ & 0.13 & $4.36 \%$ \\
\cline { 2 - 4 } & $b$ & 56.61 & $3.33 \%$ \\
\hline Kelvin-Voigt & $b$ & 14.97 & $1.98 \%$ \\
\cline { 2 - 4 } & $k$ & 104.69 & $0.44 \%$ \\
\hline $\begin{array}{c}\text { Kelvin- } \\
\text { Boltzmann }\end{array}$ & $\beta$ & 30.76 & $1.13 \%$ \\
\cline { 2 - 4 } & $\gamma$ & 100.43 & $0.33 \%$ \\
\cline { 2 - 4 } & $\alpha$ & 0.06 & $1.78 \%$ \\
\hline
\end{tabular}

Table 1: Estimated parameters for each model

In (1), the parameter $\beta$ is obtained as a combination of the coefficients of the two springs, and is related to tissue's stiffness. The parameters $\alpha$ and $\gamma$ are related to the tissue's viscosity and are responsible for the transient accuracy of the model.

\section{Model-based force control}

The force control algorithm proposed here uses the Kelvin-Boltzmann model to achieve a good disturbances rejection caused by physiological motions.

Using the linearization presented in [2] the robot motion in Cartesian space is decoupled and the robot can be interpreted as a double integrator along each axis. The robot equation is written as:

$$
\ddot{x}=u \quad(2)
$$

Where $x$ is the robot position in Cartesian space and $u$ is the control input.

Differentiate both sides of Kelvin-Boltzmann equation, we have

$$
\alpha \ddot{F}_{e}+\dot{F}_{e}=\gamma \dot{x}+\beta \ddot{x}
$$

Substituting (3) in (2) the system equation is rewritten as

$$
\alpha \ddot{F}_{e}+\dot{F}_{e}=\gamma \dot{x}+\beta\left(u+\ddot{x}_{d}\right)
$$

where, $x_{d}$ is the disturbance motion.

Then, we can choose a control law given by:

$$
u=-\ddot{x_{d}}-\frac{\gamma}{\beta}\left(\dot{x_{r}}+\dot{x_{d}}\right)-\frac{a_{1}}{\beta} \Delta F-\frac{a_{2}}{\beta} \dot{F_{e}}
$$

where $\Delta F=F_{e}-F_{d}$ and $a_{1}, a_{2}$ are positive constant control parameters. Substituting the control law in (4), we have:

$$
\alpha \Delta \ddot{F}+\left(1+a_{2}\right) \Delta \dot{F}+a_{1} \Delta F=0
$$


In order to achieve critically damping, i.e. with 0 overshot in force tracking to guarantee safety, the control parameters $a_{1}, a_{2}$ should be chosen as $a_{1}=$ $\frac{\left(1+a_{2}\right)^{2}}{4 \alpha}$. More details about the force control scheme can be seen in [8].

\subsection{Performance through Simulation Studies}

Evaluation

To evaluation the control performance of the proposed force control method and the system's capability to handle disturbance and model parameter mismatch, simulation studies have been carried out based on the D2M2 robot model with all dynamic and kinematic parameters calibrated from the real robot and using in vivo heart beating motion data recorded through Da Vinci system. The three-dimensional heart beating motion data are illustrated as in Fig 3 .
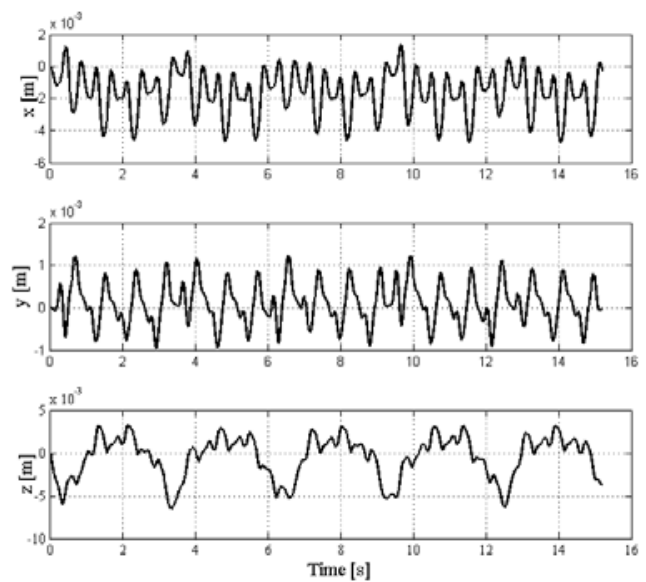

Figure 3: 3D heart motion

Since the robot end-effector just touches a specified point on the beating heart, no torsion is involved in the force measurement hence it's reasonable to assume that the force measurements for three axes are non-coupled. According to the experiments introduced in Section II, the Kelvin-Boltzmann model identified and used in the simulation study is

$$
F_{e}=100.65 x_{r}+30.705 \dot{x_{r}}-0.0567 \dot{F}_{e} .
$$

The control parameters in the robot force control input are set to $a_{1}=30, a_{2}=1$. To fully explore the capability of the proposed control method, the simulation is composed of 3 phases: $0-3$ second, a step force command with amplitude of $4 \mathrm{~N}$ is sent to the robot at time 1 second without presence of the beating heart motion disturbance yet; 3-6 second, the heart beating disturbance as in Fig 3 is introduced into the interaction force between the robot and tissue; 6-15 second, pulse series of amplitude of $4 \mathrm{~N}$ are added in the force command with the presence of heart beating disturbance. Simulation results are illustrated in Fig 4.
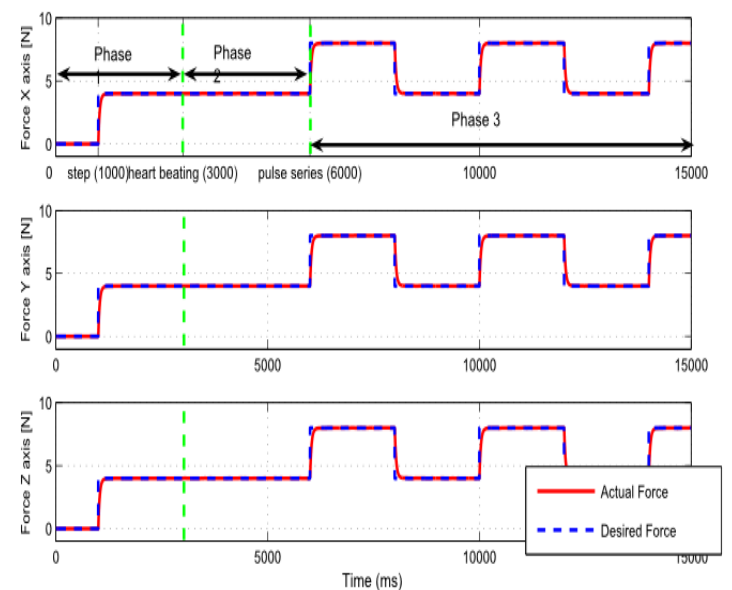

Figure 4: 3D Force Tracking Performance for X, Y, Z axis

It is seen that the contact forces along all 3 axes converge fast to desired values in different phases even with the fast heart beating disturbance. In [8] more simulations results using a similar control scheme can be seen.

\section{Conclusion}

This paper presents the work of force control design for robotic-assisted beating heart surgery based on viscoelastic interaction model. The viscoelastic model is chosen and evaluated through in vitro experiments. The stability of developed control method is analyzed by theory. Simulation studies based on real robotic system setup are conducted to confirm the effectiveness the developed control method.

For future works, first the developed control method is to be implemented for it in vitro experiments in lab tests which are currently undergoing. Then the method will be further improved by including real-time heart motion modeling techniques. In the end, it is planned to carry out in vivo experiments to test its feasibility and find out potential constraints for practical clinical applications.

\section{References}

[1] Y. C. Fung, Biomechanics: Mechanical Properties of Living Tissue, Springer, 1993.

[2] Wisama Khalil, Etienne Dombre, Modeling, identification \& control of robots, Butterworth-Heinemann, 2004 
[3] H. Paul, W. Bargar, B. Mittelstadt, B. Musits, R. Taylor, P. Kazanzides, and e. al., Development of a Surgical Robot for Cementless Total Hip Arthroplasty, Clinical Orthopaedics and Related Research, vol. 285-, pp. 57-66, Dec 1992

[4] R. Cortesão, W. Zarrad, P. Poignet, O. Company and Etienne Dombre, Haptic Control Design for RoboticAssisted Minimally Invasive Surgery, in Int. Conf. on Intelligent Robots and Systems, 2006, pp 454-459.

[5] M. Dominici, R. Cortesão and C. Souza, Heart Motion Compensation for Robotic-Assisted Surgery: Predictive Approach vs Active Observer, Proc. IEEE Int. Conf. on Robotic and Automation, 2011, pp 6252 - 6257.

[6] B. Cagneau, N. Zemiti, D. Bellot and G. Morel, Physiological Motion compensation in robotized surgery using force feedback control, Proc. IEEE Int. Conf. on Robotic and Automation, 2007, pp 1881-1886.
[7] R. Cortesão, On Kalman Active Observers, Journal of Intelligent and Robotic Systems, vol 48, Issue 2, February, 2007

[8] Chao Liu, Pedro Moreira, Nabil Zemiti and Philippe Poignet, 3D Force Control for Robotic-Assisted Beating Heart Surgery Based on Viscoelastic Tissue Model, Int. Conf. of the IEEE Engineering in Medicine and Biology Society, 2011, pp 7054-7058. 